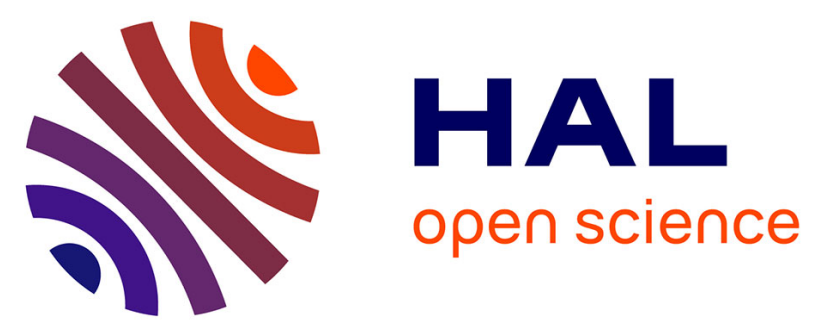

\title{
Microlens self-writing on vertical laser diodes by Near Infra-Red photo-polymerization
}

David Barat, Véronique Bardinal, I. Dika, O. Soppera, Anna Rumyantseva, Benjamin Reig, Mickaël Renault, Aurélien Bruyant, Jean-Baptiste Doucet, Thierry Camps, et al.

\section{To cite this version:}

David Barat, Véronique Bardinal, I. Dika, O. Soppera, Anna Rumyantseva, et al.. Microlens selfwriting on vertical laser diodes by Near Infra-Red photo-polymerization. Microelectronic Engineering, 2013, 111, pp.204-209. 10.1016/j.mee.2013.03.155 . hal-02569641

\section{HAL Id: hal-02569641 \\ https://hal.science/hal-02569641}

Submitted on 11 May 2020

HAL is a multi-disciplinary open access archive for the deposit and dissemination of scientific research documents, whether they are published or not. The documents may come from teaching and research institutions in France or abroad, or from public or private research centers.
L'archive ouverte pluridisciplinaire HAL, est destinée au dépôt et à la diffusion de documents scientifiques de niveau recherche, publiés ou non, émanant des établissements d'enseignement et de recherche français ou étrangers, des laboratoires publics ou privés. 


\section{Microlens self-writing on vertical laser diodes by Near Infra-Red}

\section{photo-polymerization}

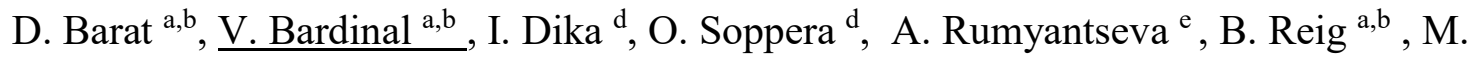
Renault $^{\mathrm{e}}$, A. Bruyant ${ }^{\mathrm{e}}$, J.B. Doucet ${ }^{\mathrm{a}, \mathrm{b}}$, T. Camps ${ }^{\mathrm{a}, \mathrm{c}}$, J..P. Malval ${ }^{\mathrm{d}}$, E. Daran ${ }^{\mathrm{a}, \mathrm{b}}$

${ }^{\text {a } C N R S ~ ; ~ L A A S ~ ; ~} 7$ avenue du colonel Roche, F-31400 Toulouse, France

${ }^{\mathrm{b}}$ Université de Toulouse, LAAS, F-31400 Toulouse, France

${ }^{\mathrm{c}}$ Université de Toulouse, UPS, LAAS, F-31400 Toulouse, France

${ }^{\mathrm{d}}$ CNRS IS2M, Institut de Science des Matériaux de Mulhouse (IS2M), CNRS - LRC 7228, Université de Haute Alsace, 15 rue Jean Starcky, Mulhouse, France

${ }^{\text {e }}$ LNIO-CNRS, Institut Charles Delaunay, Université de Technologie de Troyes, 12, rue Marie Curie - BP 2060 - 10010 Troyes Cedex, France

Keywords: micro-optics, laser writing, photo-polymerization, optical probing, maskless fabrication

e-mail : bardinal@laas.fr

Tel: (33)5 61337836

Fax: (33)5 61336208 
Abstract

We report on the improvement of a fabrication method for micro-optics integration on vertical semiconductor laser diodes (VCSELs). Our approach is based on microlens self-writing by the laser beam itself thanks to self-guided Near-Infra-Red photopolymerization. Fabrication conditions have been optimized to control and take benefit of process sensitivity to oxygen quenching and to make possible the study of photo-chemical parameters influence on final tip geometry. The interest of this simple method for fabricating focused-beam VCSELs is finally discussed.

\section{Introduction}

Due to a high parallel operation and a low power consumption, VCSELs (Vertical-Cavity Surface-Emitting Lasers) have become laser sources of great importance for optical interconnects and for compact optical sensors [1-2]. To be exploited in such systems, VCSELs are associated to external micro-optical elements to adapt their natural far-field beam divergence (from $10^{\circ}$ to $22^{\circ}$ half angle at $1 / \mathrm{e}^{2}$, depending on fabrication technology) to the aimed application. Consequently, VCSEL beam shaping using collective and simple methods is strongly in demand [3]. In particular, beam focusing up to the diffraction limit stays a key issue for exploiting singlemode VCSELs for sensing, measurement or printing [45]. With this aim, we have developed a new method for polymer microtips self-writing on GaAs-based VCSELs using self-guided Near Infra-Red (NIR) photo-polymerization. This approach implements the use of photopolymers that are sensitive at the VCSEL lasing wavelength. In these conditions, the fabrication process of polymer microlens at the laser output is triggered by the laser source itself and can be applied to devices arrays during their collective test under probes, without the need for a lithographic step. The method feasability 
was first demonstrated using a local dispending technique [6]. More recently, we investigated the properties of $760 \mathrm{~nm}$ tipped-VCSELs with different geometries, by playing on photochemical parameters [7]. This study was possible thanks to the development and to the optimization of a wafer level fabrication process that we detail in this paper.

\section{Experimental methods}

\subsection{NIR self-writing on VCSELs}

Our method is derivated from a simple and low cost technique earlier proposed in the visible spectral range for implementing a polymer micro-component at the extremity of an optical fiber. A drop of photopolymer is deposited at the end of a fiber in which a green laser light is coupled [8]. Light exposure initiates a photopolymerization process and a self-guiding effect occurs thanks to a change in the refractive index of the photopolymer upon irradiation. After a standard developing step, a self-aligned microtip remains at the surface of the fiber and allows for a strong focusing of the output beam. This effect can be exploited for nearfield optical microscopy or scanning probe microscopy applications.

To apply this method to VCSELs devices, several conditions must be met: first, the dye has to absorb light in the Near-Infra-Red spectrum and initiate polymerization, since this range corresponds to the typical emission range of most advanced VCSELs, i.e. in GaAs technology $(700 \mathrm{~nm}-1 \mu \mathrm{m})$. Secondly, the resist has to be totally bleached upon irradiation so that it becomes transparent after fabrication. Indeed, for VCSEL case, the operating wavelength will be indeed inside the actinic polymerization wavelength span. Furthermore, the resist has to be easily dispended on the VCSELs wafer. Finally, laser operation should be minimally disturbed by microtip fabrication so that main VCSEL features (modal behaviour, low threshold, emitted power) are kept. To fulfill these requirements, we have developed new chemical systems based on free radical photopolymerization. Our formulation is a mixture of 
three components: a NIR sensitizer, a cyanine (HITC), associated with a co-initiator (MDEA) to trigger the polymerization of a triacrylic monomer (PETIA). Such a monomer presents good performances in terms of spatial resolution and leads to a crosslinked polymer with suitable mechanical and optical properties for integrated micro-optics on laser devices. The molecular structure of the different components is shown in Fig. 1, as well as the formulation transmission coefficient spectrum before and after irradiation. As required, it presents a maximum value in the NIR region, and is totally bleached after fabrication. The thickness of the inhibition layer can be controlled by NIR light intensity. Indeed, the origin of the inhibition layer is due to the continual replenishment of oxygen by diffusion from the surrounding atmosphere that does not allow reaching an oxygen concentration low enough to efficiently start the polymerization reaction. Increasing the light power creates more reactive species that consume more Oxygen and the result is a thinner inhibition layer. A more detailed description of involved physical and chemical phenomena can be found in [11]. More details on photochemistry properties can be found elsewhere [9][10].

\subsection{Effect of initial thickness of the film}

Figure 2 illustrates the prominent role of initial film thickness on the geometry of fabricated microtips on VCSELs devices. Indeed, the initial film thickness not only governs the final tip height (because, obviously, the size of the tip cannot exceed the film thickness), but also the shape of the tip. In fact, the overall process is ruled by light-driven processes (photo-polymerization) but also by oxygen inhibition. The thickness of the inhibition layer can be controlled by NIR light intensity. Indeed, the origin of the inhibition layer is due to the continual replenishment of oxygen by diffusion from the surrounding atmosphere that does not allow reaching an oxygen concentration low enough to efficiently start the polymerization reaction. Increasing the light power creates more reactive species that consume more Oxygen 
and the result is a thinner inhibition layer. A more detailed description of involved physical and chemical phenomena can be found in [11]. The consequence of oxygen inhibition and of diffusion from the surface is the presence of a thin layer at the surface of the film in which polymerization is not efficient. Figure 2 illustrates what happened for 3 different thicknesses ranging from 5 to 60 microns. In all cases, we observed that the thickness of the tip was smaller than the initial film thickness. The difference was, for the light intensity that was chosen, around 5 microns. For the thinnest film ( 5 microns), the tip could hardly be observed. Indeed, in this case, the thickness of the film is of the same order of magnitude of the inhibition thickness. For the thicker layer $(60 \mu \mathrm{m})$, polymerization proceeds as well up to the limit of inhibition but in this case, the aspect ratio of the tip exceeds the maximum acceptable value for the mechanical properties of this material. The consequence is a tip with a twisted end, unsuitable for applications. In consequence, the thickness range suitable for the tip fabrication is comprised between 20 and 50 microns. Only in this range, it is possible to obtain straight tips and to tune their end shape in a wide range of radius of curvature, by changing the photonic parameters (optical power and exposure time).

\subsection{Film deposition using spin-coating}

First experiments on VCSELs were performed using a local dispensing method for NIR resist deposition [6]. Tip creation was demonstrated with VCSELs emitting just above their laser threshold. However, the precise control of resist thickness in the above-mentioned required range $(20-50 \mu \mathrm{m})$ was not possible and tip shape was thus not reproducible. To solve this issue, a spin-coating process was developed. As seen in Fig. 3, this method present other advantages, as it does not require an alignment of the droplet relatively to the emitting device and it is more suited for a collective fabrication at a wafer-scale. Viscosity of the photopolymer has been adjusted by a judicious choice of monomers to reach the targeted thickness value and to present a behavior similar to the one of a commercial negative tone 
resist (SU-3025). In parallel, substrate functionalization has been proceeded in order to guarantee an optimal anchoring of the polymer on the VCSEL surface and a good surface quality. Thickness profile after spinning and polymerization on a 2" diameter wafer was characterized by means of a mechanical profilometer for two different spinning speeds (1000 and $2000 \mathrm{rpm}$, during 30s, acceleration: $5000 \mathrm{pms}^{-1}$ ) (Fig. 4). In both cases, the targeted thickness was reached $(48 \mu \mathrm{m}$ and $24 \mu \mathrm{m})$ and uniformity was found to be better than $2 \mu \mathrm{m}$ on the whole surface, except in a ring-shape area located at the wafer edges $(2.5 \mathrm{~mm})$. This is due to a typical material built-up occurring during spin coating. This edge-bead could be removed but this is not necessary since in our case no mask will be used for photo-fabrication.

\subsection{Photofabrication under optical control}

After NIR formulation deposition, photofabrication was performed using a simple electrical probe station commonly used for L-I-V (Light-Intensity-Voltage) measurements of vertical laser diodes emission properties. Thanks to an optical microscope, a metallic microprobe was placed on the VCSEL top contact pad (anode) to allow for an electrical injection. Precise probe positioning was possible thanks to formulation transparency in the visible range (see Fig. 1). As illustrated in Fig. 5, a silicon photodiode was also placed above the emitting device to measure the light transmitted through the polymer during fabrication. This real-time control allowed for the control of the end of the reaction by checking formulation transparency. Bleaching time was thus found to vary from $4 \mathrm{~s}$ to $16 \mathrm{~s}$ depending on the initial VCSEL optical power. The signal was also acquired as a function of the optical dose for different VCSEL biasing conditions, i.e. different initial emitted powers (Fig. 5). The normalized curves saturate for the same value of optical dose $\left(40 \mathrm{~s} . \mathrm{kW} / \mathrm{cm}^{2}\right)$. This means that the photo-fabrication mechanism is not dependant of optical power level and that in this range of incident power tip geometry can be tuned by playing only on the exposure dose. 


\section{Parametric study and discussion}

The influence of chemical and opto-electrical parameters was investigated for two different types of singlemode VCSELs devices presenting two different emission sizes $(5 \mu \mathrm{m}$ and $3 . \mu \mathrm{m}$ with beam divergences of $17^{\circ}$ and $22^{\circ}$, respectively). Figure 6 shows a parametric study carried out for VCSELs fabricated with a $5 \mu \mathrm{m}$-diameter metal aperture and for an initial resist thickness comprised between 45 and 50 microns. For this series of experiments, the dye content was chosen to be $0,09 \%$ of HITC. The VCSEL emission powers were chosen between 40 and $350 \mu \mathrm{W}$ and resist irradiation times between 2 and $600 \mathrm{~s}$. Note that missing data in the table correspond to samples that were damaged before SEM characterizations. For the lowest power $(40 \mu \mathrm{W})$ the extent of polymerization remains very weak. This is due to the inhibition and diffusion of oxygen that stop the polymerization in the film. Indeed, the thickness of inhibition described in the previous figure is directly dependant on the used power. Low power favors the influence of the diffusion of oxygen as it allows a continuous replenishment of oxygen from the surface. Under such conditions, the inhibition can never be overcome by polymerization, resulting in a saturation of the tip height to very low value. Interestingly, this gives an evaluation of the inhibition thickness for such power, which can be estimated to 40 microns. When the power is increased to 170 and $280 \mu \mathrm{W}$, the extent of polymerization is increased and resulting tips are higher, as expected. Interestingly, these values of power correspond to a wide dynamic range in tips geometry, which is obviously the regime of interest for future applications since the tips properties can be adjusted in a wide range by playing on photonic parameters. Finally, the use of higher powers $(350 \mu \mathrm{W})$ showed low interest since in this case, the polymerized volume can be hardly controlled and resulting tips exhibit irregular shapes.

The previous results were confirmed with a second series of experiments carried out on a second family of VCSEL (namely "oxide-confined" VCSELs that exhibit a smaller 
emission zone of $3 \mu \mathrm{m}$, as well as a more divergent beam) (Fig. 7). In this case, the dye concentration was $0.18 \%$ and the resist thickness was kept between 45 and 50 microns. Though these results cannot be directly compared since the emission surface is different from the 2 series of VCSELs, the same trends were observed. Indeed, for the lowest power (40 $\mu \mathrm{W}$ ), we observed a limited size of the microtip (less than 5 microns in height), which again can be explained by the inhibition layer at the surface of the photopolymer. When the power was increased, the tip height was found to increase and to saturate around 30 microns for powers greater than $100 \mu \mathrm{W}$. Interestingly, it was observed that tuning the tip height was more complicated as for the previous VCSEL devices. For the lowest irradiation times (2s), the tips had the same length than for highest irradiation but resulted in poorly cross-linked polymer with low mechanical properties. The optimal fabrication parameters appear thus around $60 \mu \mathrm{W}$, for which straight tips with tunable length and radius of curvature at the extremity can be reached. These results shows that self-guided polymerization remains possible even in the case of large numerical aperture VCSEL such as oxide-confined VCSELs. As for the discrepancies observed on tip shapes and tip heights between the two types of devices, they can be mainly accounted for the differences of initial beam profiles [7].

Once optimal fabrication conditions are defined, typical dimension ranges of microtips fabricated at the surface of NIR single-mode VCSELs were extracted (Table 1). Tip ROC (Radius of Curvature), tip height and tip basis diameter were found to increase with the exposure dose. Highest values were indeed obtained in the saturation regime, when the actinic light reaches the inhibition zone, leading to longer, larger and flatter tips. Note that the ROC range could be further extended by depositing an intermediate transparent layer between the VCSEL and the NIR formulation (to increase the beam size before reaction) and/or by modifying the chemical composition of the formulation. Taking into account these experimental data, focusing properties of tipped- VCSELs were modeled using a FDTD 
(Finite-Difference Time-Domain) method, as illustrated in Fig. 8. Our simulations indicated that tip insertion on VCSEL leads to a strong beam focusing near the tip surface (working distance $<1 \mu \mathrm{m})$. These trends were successfully compared to experimental measurements performed on mounted devices using a high numerical aperture objective for spot imaging [7]. Spot diameters as low as $500 \mathrm{~nm}$ could be measured near the tip surface for the sharpest tips. It is worth noting that other VCSELs features (lasing wavelength, modal behavior) were not significantly disturbed by tip presence, except regarding the laser threshold that was slightly increased due to a modification of VCSEL top reflectivity. This optical study is still undergoing. Nevertheless, the ability of our technique to fabricate collectively focused-beam VCSELs sources has been demonstrated.

\section{Conclusions}

We have presented a NIR self-writing process suited for the integration of self-aligned microtips on vertical cavity laser devices (VCSELs). This single-step and room temperature technique is based on the use of NIR photopolymers that were optimized for spin coating to make possible a precise control of initial thickness prior to photofabrication. As a result, limitation of oxygen quenching effects was possible as well as a collective fabrication at a wafer-scale. Thanks to this improvement, a parametric study was also led on two different kinds of VCSEL devices. Suitable exposure doses and corresponding dimensions ranges were determined. The focusing abilities of such tipped-devices were also evaluated, showing that our method could be exploited for the fabrication of compact optical micro-probes for scanning probe microscopy or for near-field optical microscopy with an optimization of the polymer probe [12]. 
Acknowledgements

The authors gratefully acknowledge French National Research Agency (ANR) for financial support (ANR-09-BLAN-0168-01 and RENATECH (French Network of Major Technology Centres) within LAAS-CNRS for technological support. The authors would also like to thank Oclaro Inc. (Zürich, Switzerland) and Philips Technology GmbH U-L-M Photonics (Ulm, Germany) for providing 760nm-VCSEL devices. 


\section{References}

[1] K. Iga, « Vertical-Cavity Surface-emitting Laser : its conception and evolution » Japanese Journal of Applied Physics Vol.47 №1 pp1-10 (2008)

[2] A. Larsson "Advances in VCSELs for Communication and Sensing" Selected Topics in Quantum Electronics, IEEE Journal of , vol.17, no.6, pp.1552-1567, Nov.-Dec. 2011

[3] V. Bardinal, T. Camps, B. Reig, D. Barat, E. Daran, and J.-B. Doucet "Collective micro-optics technologies for VCSEL photonic integration" Advances in Optical Technologies, vol. 2011, 609643, 2011.

[4] D. Heinis, C. Gorecki, C. Bringer, V. Bardinal, T. Camps, J.B. Doucet, P. Dubreuil and C. Fontaine "Miniaturized scanning near-field microscope sensor based on optical feedback inside a single-mode oxide-confined Vertical-Cavity Surface-Emitting Laser,” Jpn. J. Appl. Phys. 42, Part2, N¹2A, 1469-1471 (2003).

[5] N. Mukoyama, H. Otoma, J. Sakurai, N. Ueki, and H. Nakayama, "VCSEL array based light exposure system for laser printing," Proc.SPIE, vol. 6908, pp. 69080H-1-69080H11, Feb. 2008

[6] V. Bardinal, B. Reig, T. Camps, E. Daran, J.B. Doucet, C. Vergnenègre, C. Turck, J.P. Malval, D.J. Lougnot, O. Soppera, “A microtip self-written on a Vertical-Cavity SurfaceEmitting Laser by photopolymerization,” Appl. Phys. Lett. 96, 051114 (2010).

[7] D. Barat, V. Bardinal, I. Dika, O. Soppera, P. Debernardi, A. Rumyantseva, B. Reig, M. Renault, T. Camps, A. Bruyant, J.B. Doucet, J.P. Malval, and E. Daran, "Photo-chemical study and optical properties of microtips self- written on vertical laser diodes using NIR photo-polymerization," Opt. Express 20, 22922-22933 (2012)

[8] R. Bachelot, P. Royer, G. Wurtz, C. Ecoffet, A. Espanet, D.J. Lougnot, French Patent N$^{\circ}$ PCT 9814385, (2001). 
[9] O. Soppera, C. Turck, D.J. Lougnot, "Fabrication of micro-optical devices by self-guiding photopolymerization in the near IR," Opt. Lett. 34(4), 461-463 (2009).

[10]I. Dika, J.P. Malval, O. Soppera, V. Bardinal, D. Barat, C. Turck, A. Spangenberg, A. Bruyant, "Near-infrared photopolymerization: initiation process assisted by selfquenching and triplet-triplet annihilation of excited cyanine dyes," Chem. Phys. Lett. $515,1-3,91-95(2011)$.

[11]O. Soppera, S. Jradi, D.J. Lougnot, "Photopolymerization with microscale resolution : influence of the physico-chemical and photonic parameters" J. Polym. Sci., Part A : Polym. Chem., 46(11), 3783-3794 (2008)

[12]Z. Sedaghat, A. Rumyantseva, A. Bruyant, S. Kostcheev, S. Blaize, S. Jradi, R. Bachelot, A. Monmayrant,"Near-field optical imaging with a nanotip grown on fibered polymer microlens," Appl. Phys. Lett.100(3), 033107 (2012). 
Figure captions

Fig.1. Molecular structure of the formulation chosen for self-aligned photo-fabrication on NIR-VCSELs and corresponding transmission spectrum before (blue curve) and after (red curve) irradiation.

Fig.2 Role of initial film thickness on the final shape of fabricated microtips: the inhibition zone due to oxygen quenching prevents optimal fabrication if the layer is too thin $(5 \mu \mathrm{m})$. On the other hand, for layer that are too thick $(60 \mu \mathrm{m})$ the tip aspect ratio is too high to lead to a symetric shape.

Fig.3 Comparison of local dispending (left) and spin-coating (right) methods for NIRsensitive formulation deposition on VCSEL wafers.

Fig.4 : Thickness profiles measured after spin coating and photopolymerisation on a 2" wafer for two different spinning speeds (1000 and $2000 \mathrm{rpm})$.

Fig.5: (left) Principle of microtip NIR self-writing on VCSELs under real-time optical control. (right) Normalized optical signals measured as a function of optical dose and for different VCSEL emitted powers.

Fig. 6. SEM (Scanning Electronic Microscopy) images of microtips self-written at the surface of VCSEL devices (metallic aperture) for increasing exposure times and increasing initial VCSEL powers. 
Fig. 7. SEM (Scanning Electronic Microscopy) images of microtips self-written at the surface of VCSEL devices (oxide aperture) for increasing exposure times and increasing initial VCSEL powers.

Table 1: Possible dimensions ranges for tip ROC, tip height and tip basis diameter of microtips fabricated on singlemode oxide confined VCSELs (emission zone $3 \mu \mathrm{m}$ ).

Fig.8. (top) Description of main parameters used for tipped-VCSELs optical simulation and corresponding optical micrographs of a mounted device. (bottom) FDTD simulation result of tip focusing effect and corresponding optical micrographs of a mounted device under operation. 
Fig.1

\section{Photoinitiator}
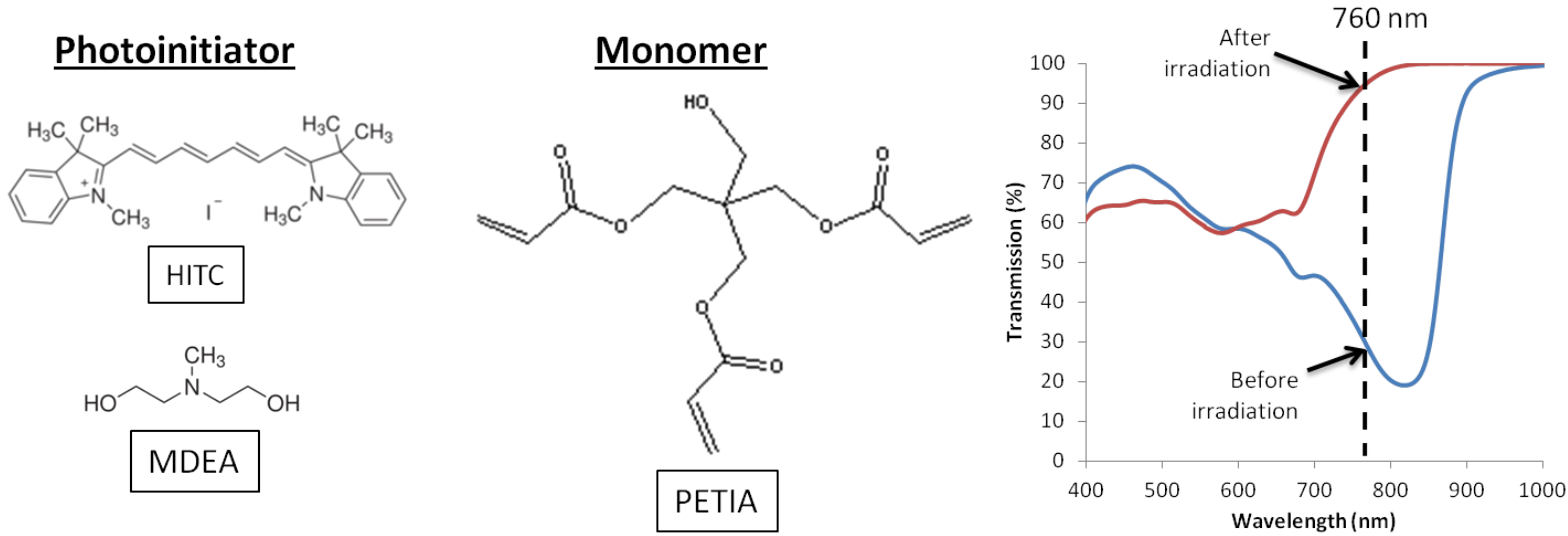
Fig. 2
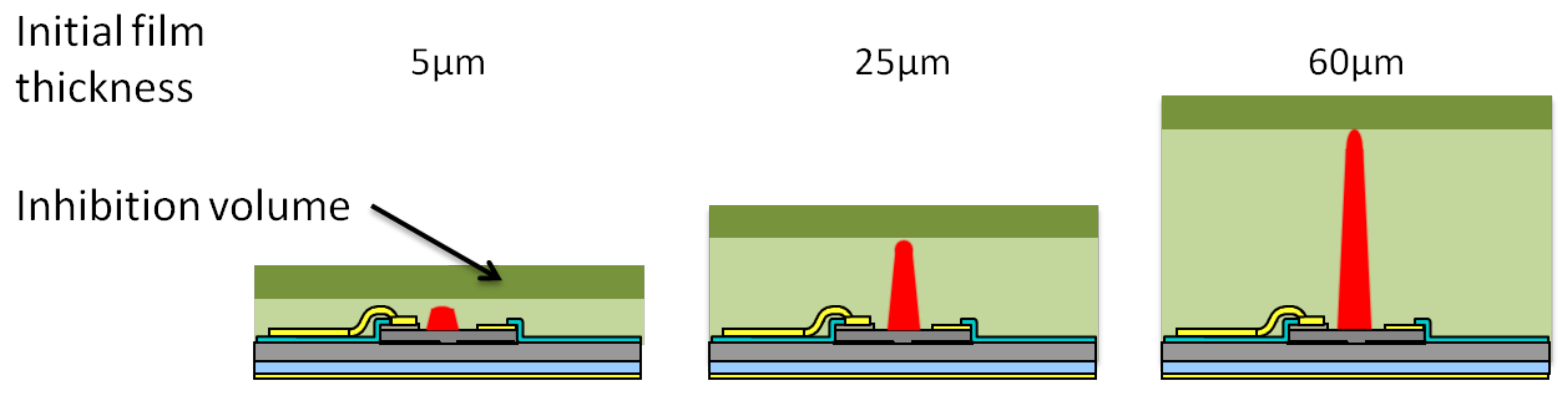

Resulting

microtip
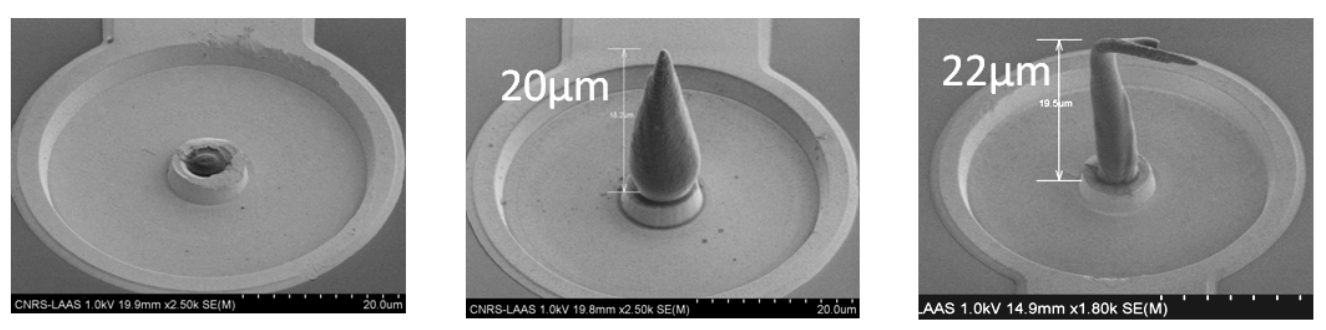
Fig.3

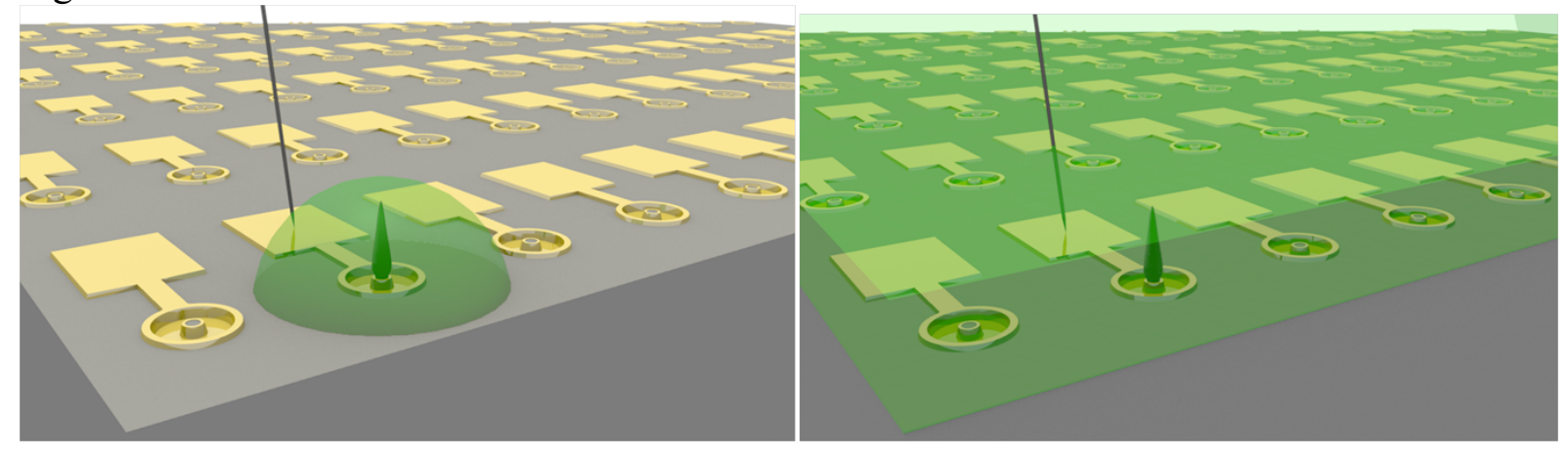


Fig.4

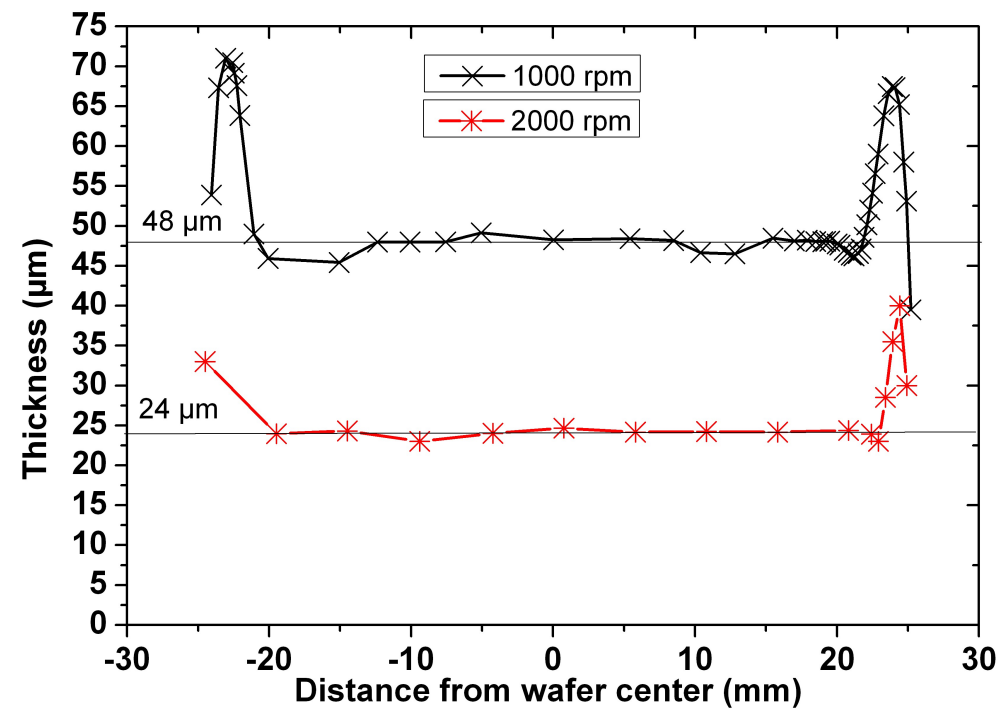


Fig. 5
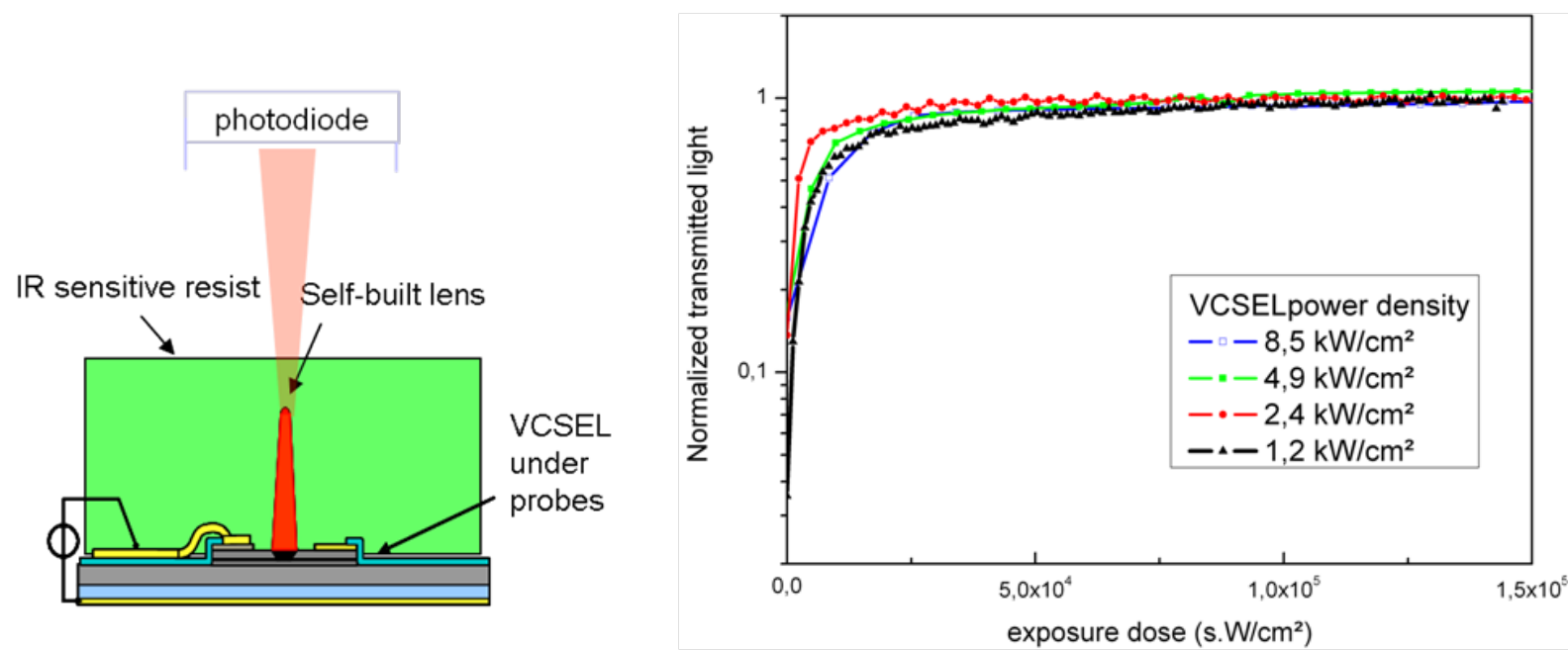
Fig.6

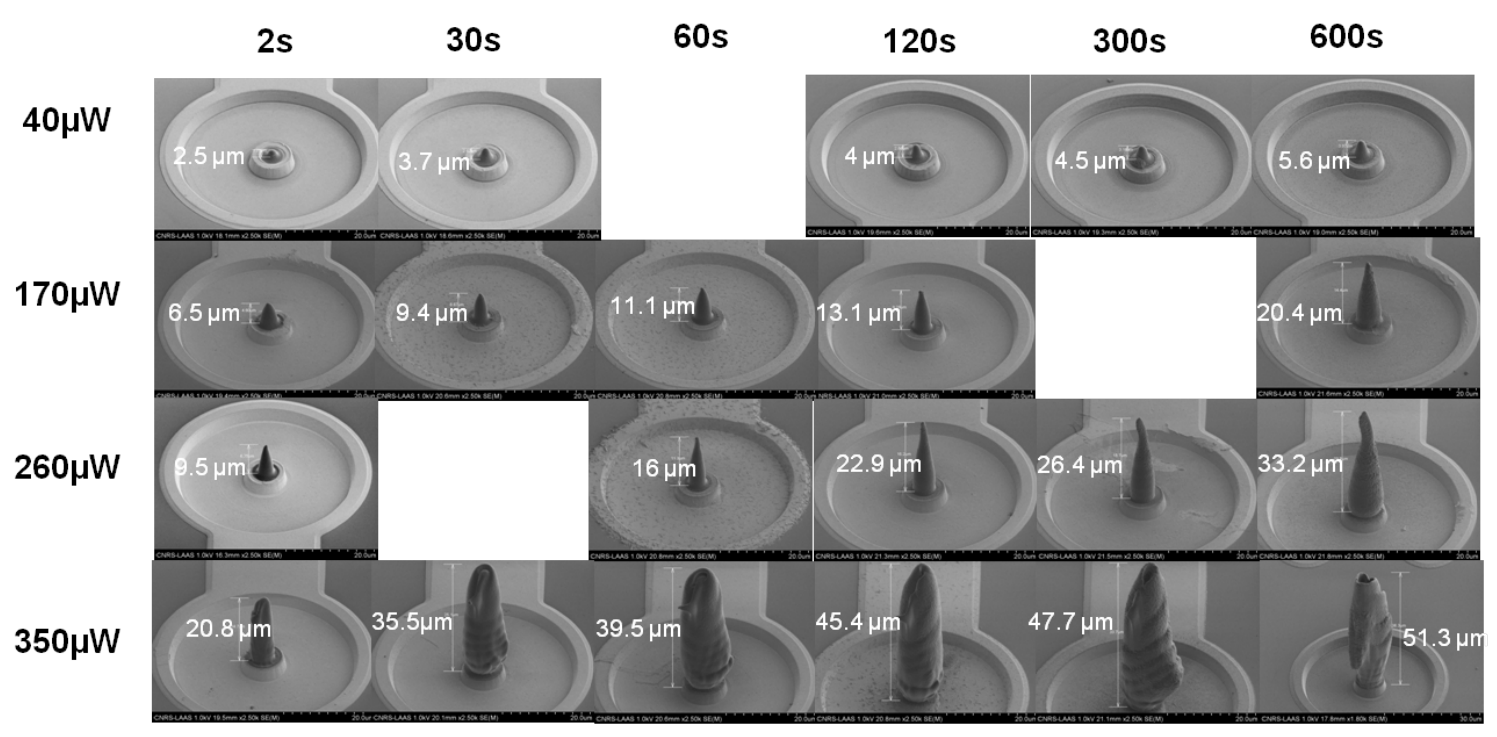


Fig. 7

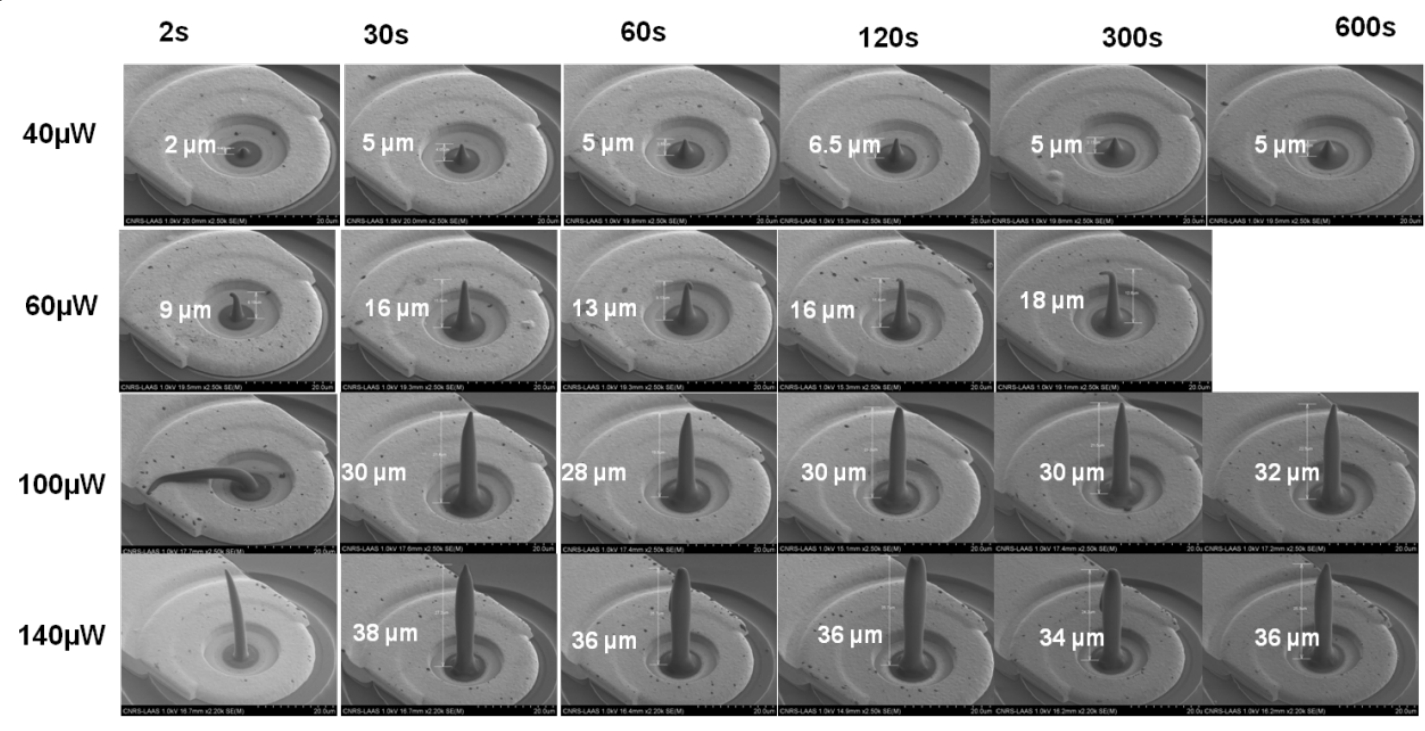


Table 1

\begin{tabular}{|c|c|c|}
\hline & Min. value $(\boldsymbol{\mu m})$ & Max. value $(\boldsymbol{\mu m})$ \\
\hline Tip ROC & 0.4 & 2 \\
\hline Tip Length & 10 & 40 \\
\hline Tip base diameter & 3 & 5 \\
\hline
\end{tabular}


Fig. 8
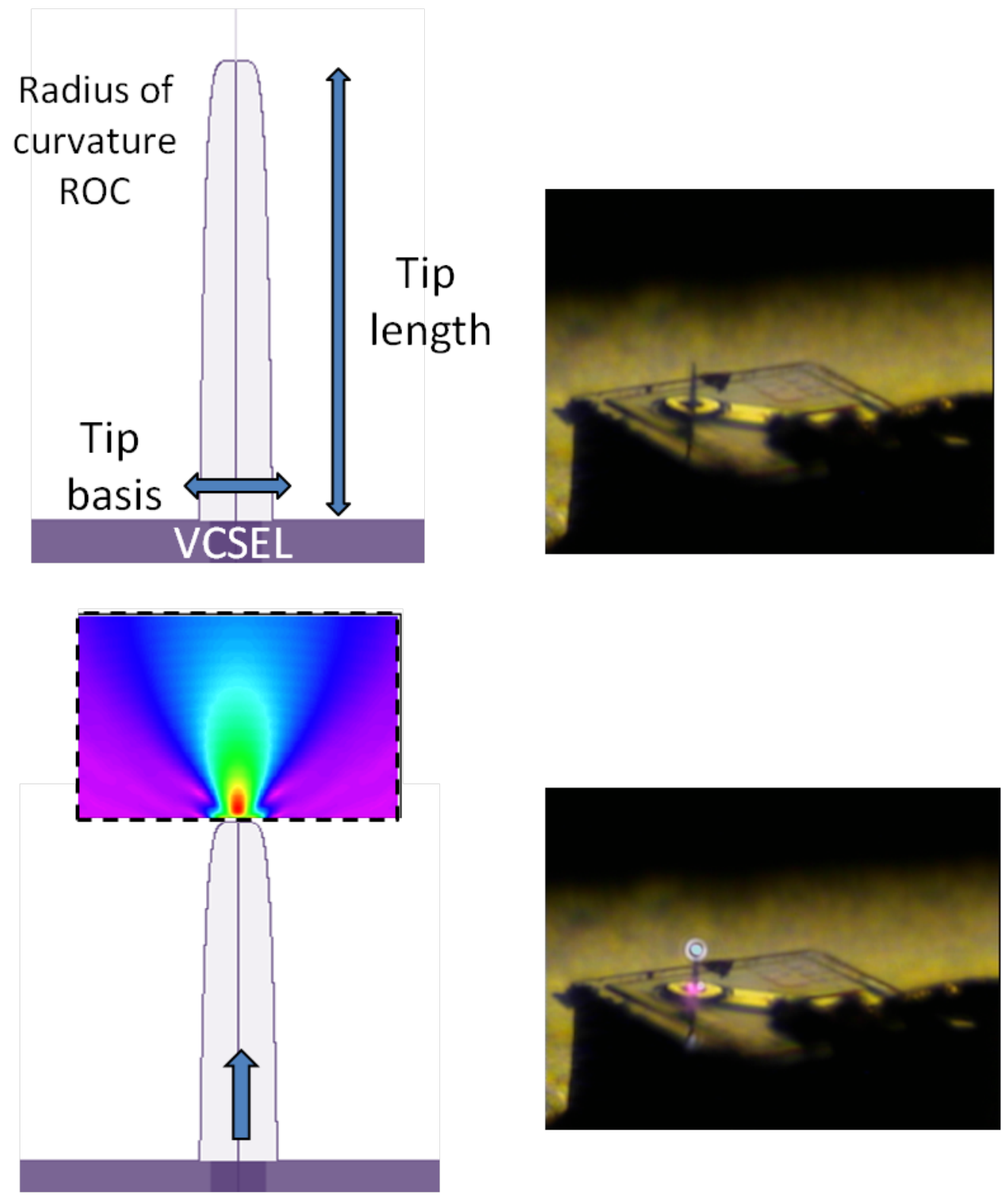